\title{
Andrographolide protects mouse astrocytes against hypoxia injury by promoting autophagy and S100B expression
}

\author{
Juan $\mathrm{Du}^{1 *}$, Chunyan Zhang ${ }^{1 *}$, Xueqing $\mathrm{Na}^{2}$, Aizhi $\mathrm{Li}^{1}$, Qingfeng Zhang ${ }^{1}$, Kezhong $\mathrm{Li}^{1}$ \\ and Yongbo Ding ${ }^{1,3}$ \\ ${ }^{1}$ Department of Anesthesiology, The Affiliated Yantai Yuhuangding Hospital of Qingdao University, Yantai, China \\ ${ }^{2}$ Department of Anesthesiology, The Second Affiliated Hospital of Kunming Medical University, Kunming, China \\ ${ }^{3}$ Department of Anesthesiology, School of Medicine, Shandong University, Jinan, China
}

\begin{abstract}
Andrographolide (ANDRO) has been studied for its immunomodulation, anti-inflammatory, and neuroprotection effects. Because brain hypoxia is the most common factor of secondary brain injury after traumatic brain injury, we studied the role and possible mechanism of ANDRO in this process using hypoxia-injured astrocytes. Mouse cortical astrocytes C8-D1A (astrocyte type I clone from C57/BL6 strains) were subjected to 3 and $21 \%$ of $\mathrm{O}_{2}$ for various times $(0-12 \mathrm{~h})$ to establish an astrocyte hypoxia injury model in vitro. After hypoxia and ANDRO administration, the changes in cell viability and apoptosis were assessed using CCK-8 and flow cytometry. Expression changes in apoptosis-related proteins, autophagy-related proteins, main factors of JNK pathway, ATG5, and S100B were determined by western blot. Hypoxia remarkably damaged C8-D1A cells evidenced by reduction of cell viability and induction of apoptosis. Hypoxia also induced autophagy and overproduction of S100B. ANDRO reduced cell apoptosis and promoted cell autophagy and S100B expression. After ANDRO administration, autophagy-related proteins, S-100B, JNK pathway proteins, and ATG5 were all upregulated, while autophagy-related proteins and s100b were downregulated when the jnk pathway was inhibited or ATG5 was knocked down. ANDRO conferred a survival advantage to hypoxia-injured astrocytes by reducing cell apoptosis and promoting autophagy and s100b expression. Furthermore, the promotion of autophagy and s100b expression by ANDRO was via activation of jnk pathway and regulation of ATG5.
\end{abstract}

Key words: Andrographolide; Hypoxia-injured astrocytes; JNK pathway; S100B; ATG5

\section{Introduction}

Traumatic brain injury (TBI) is a major cause of death or disability worldwide, especially in young people. After severe $\mathrm{TBI}$, many people are killed by secondary injury, which is a complex set of cellular processes following trauma (1). These secondary processes can worsen the damage and account for the greatest number of TBI deaths (2). Brain hypoxia is a common cause of secondary injury. Brain hypoxia initiates a sequence of biochemical events to induce neuronal cell apoptosis, resulting in a hypoxic brain injury and dysfunction (3). Thus, brain hypoxia can aggravate TBI and is regarded as an independent predictor or a marker of disease severity (4).

Andrographis paniculata is a medicinal herb that is widely used in China and other parts of Asia for the treatment of upper respiratory tract infections, fever, and diarrhea $(5,6)$. Andrographolide (ANDRO) is the major active component isolated from the stem and leaves of $A$. paniculata and is a natural diterpenoid lactone (7). ANDRO has been studied for its various bioactivities including immunomodulation (8), anti-inflammatory (9), anti-cancer (10), anti-viral (11), anti-bacterial (12), anti-hyperglycemic (13), and neuroprotective (14). The effect of ANDRO on cell apoptosis is complex. ANDRO protects thymocytes or endothelial cells against apoptosis $(15,16)$. On the other hand, some reports showed that ANDRO could induce apoptosis in human prostatic adenocarcinoma PC-3 cells (17) or other cancer cells (18). In addition, a previous study has showed that ANDRO could protect rat cardiomyocytes against hypoxia and reoxygenation injury (19). Furthermore, ANDRO was reported to protect mice against hypoxia/ischemia-induced oxidative brain injury (20). Therefore, we aimed to investigate whether ANDRO

Correspondence: Yongbo Ding: <dybyt@163.com>

*These authors contributed equally to this study. 
has an effect on mouse hypoxia-injured brain cells, which is still unknown.

Astrocytes are the most widely distributed class of cells in mammalian brain. These cells perform many functions, including support of endothelial cells, fueling neurons, maintenance of extracellular ion balance, nervous repair, and scarring of the brain following traumatic injuries (21-23). Therefore, we constructed a model of hypoxia injury in mouse astrocytes to investigate the role of ANDRO. To achieve this goal, we revealed the role of ANDRO from three aspects: cell apoptosis, autophagy, and S100B expression.

\section{Material and Methods}

\section{Cell culture}

Mouse cortical astrocytes C8-D1A (astrocyte type I clone from C57/BL6 strains), were purchased from the American Type Culture Collection (ATCC, CRL-2541 ${ }^{\mathrm{TM}}$, USA). Cells were cultured with high glucose Dulbecco's modified eagle medium (DMEM, Invitrogen, CA) containing $10 \%$ fetal bovine serum (FBS, Gibco, USA), $50 \mathrm{lU} / \mathrm{mL}$ penicillin G (Gibco, USA) and $50 \mu \mathrm{g} / \mathrm{mL}$ streptomycin (Gibco). Then, cells were incubated at $37^{\circ} \mathrm{C}$ in a moist atmosphere containing $5 \% \mathrm{CO}_{2}$.

\section{Hypoxia exposure}

To establish an astrocytes hypoxia injury model, cells were subjected to hypoxic $\left(3 \% \mathrm{O}_{2}, 5 \% \mathrm{CO}_{2}\right.$ balanced with $\mathrm{N}_{2}$ ) culture. Cells cultured in normoxia (room air, $5 \% \mathrm{CO}_{2}$ ) were the control.

\section{ANDRO treatment}

ANDRO was purchased from Sigma-Aldrich (365645, USA) with purity $\geqslant 98 \%$. A stock solution with a concentration of $15 \mathrm{mM}$ was prepared by dissolving ANDRO in dimethyl sulphoxide (Sigma-Aldrich, USA). ANDRO in a concentration of $10 \mu \mathrm{M}$ was used to treat cells throughout the hypoxia exposure. SP600125, an inhibitor of JNK, was purchased from Sigma (S5567, USA). SP600125 in a concentration of $10 \mu \mathrm{M}$ was used to treat cells throughout the hypoxia exposure.

\section{Viability assay}

Cells were seeded on a 96-well plate with 5000 cells/ well. Cell viability was measured by the Cell Counting Kit8 (CCK-8, Dojindo Molecular Technologies, USA). After incubation for $0-12 \mathrm{~h}$ at $37^{\circ} \mathrm{C}$, cells from control, hypoxia, and hypoxia (12 h) + ANDRO groups were added to CCK8 solution with $10 \mu \mathrm{L} /$ well and were incubated for $4 \mathrm{~h}$ at $37^{\circ} \mathrm{C}$. Then, the absorbance was measured at $450 \mathrm{nM}$ using a Microplate Reader (Bio-Rad, USA).

\section{Apoptosis assay \\ Cells for apoptosis detection were washed twice with cold PBS and resuspended in buffer. Then, cells}

were dyed using Annexin V-FITC/PI apoptosis detection kit (Invitrogen, USA) according to the manufacturer's instruction. After reaction in dark at room temperature for $10 \mathrm{~min}$, flow cytometry analysis was done by using a FACScan flow cytometer (Beckton Dickinson, USA) to differentiate apoptotic cells (Annexin- $V$ positive and $\mathrm{PI}$ negative) from necrotic cells (Annexin- $\mathrm{V}$ and $\mathrm{PI}$ positive).

\section{Cell transfection}

siRNA targeted against ATG5 (si-ATG5) and nontargeting siRNA (si-NC) used as negative control were synthesized from GenePharma Co. (China). Cell transfections were conducted using Lipofectamine 3000 reagent (Invitrogen, USA) following the manufacturer's protocol. After $48 \mathrm{~h}$ of transfection, cells were collected for further analysis.

\section{Real-time quantitative reverse transcriptase PCR (qRT-PCR)}

Total RNA was isolated from treated cells by using TRIzol reagent (Invitrogen, USA). Reverse transcription was performed by using the Prime Script RT reagent Kit (TaKaRa, China) according to the manufacturer's instructions. A SYBR Fast qPCR Mix (TaKaRa) was used to quantify the mRNA levels according to the manufacturer's instructions. $\beta$-actin was used as the internal standard. The ${ }^{{ }^{\Delta}} \mathrm{Ct}$ method was used to calculate changes in expression.

\section{Western blot}

The proteins used for western blot were extracted using RIPA lysis and extraction buffer (Thermo Scientific, USA). The proteins were quantified using the BCA Protein Assay Kit (Thermo Scientific) and were separated by a NativePAGE Novex Bis-Tris Gel system (Invitrogen) according to the manufacturer's instructions. Then proteins were transferred to a Polyvinylidene Difluoride (PVDF) membrane (Millipore, USA). The membrane was incubated with primary antibodies at $4^{\circ} \mathrm{C}$ overnight, followed by washing and incubation with secondary antibody marked by horseradish peroxidase at room temperature. Primary antibodies included anti-Bcl-2 (ab59348, 1:1000; Abcam, UK), anti-Bax (ab182733, 1:2000; Abcam), anti-procaspase-3 (ab44976, 1:500; Abcam), anti-cleaved-caspase3 (ab13847, 1:500; Abcam), anti-caspase-9 (ab202068, 1:2000; Abcam), anti-LC3B (ab51520, 1:3000, Abcam), anti-Beclin-1 (ab62557, 1:1000; Abcam), anti-p62 (ab56416, $1: 1000$; Abcam), anti- $\beta$-actin (ab8224, 1:1000; Abcam), antiS100B (ab52642, 1:1000; Abcam), anti-JNK (ab179461, 1:1000; Abcam), anti-p-JNK (ab124956, 1:5000; Abcam), anti-c-Jun (ab32137, 1:5000; Abcam), anti-p-c-Jun (ab32385, 1:5000; Abcam), and anti-ATG5 (ab108327, 1:5000; Abcam). After adding ECL Plus Western Blotting Substrate (Thermo Scientific) to cover the membrane surface, the signals were captured and the intensity of the bands was quantified 
using the ChemiDoc ${ }^{\mathrm{TM}}$ XRS system (Bio-Rad, USA). $\beta$-actin antibody (Abcam) was used as the endogenous protein for reference.

\section{Statistical analysis}

All experiments were repeated three times. The results of multiple experiments are reported as means $\pm S D$.
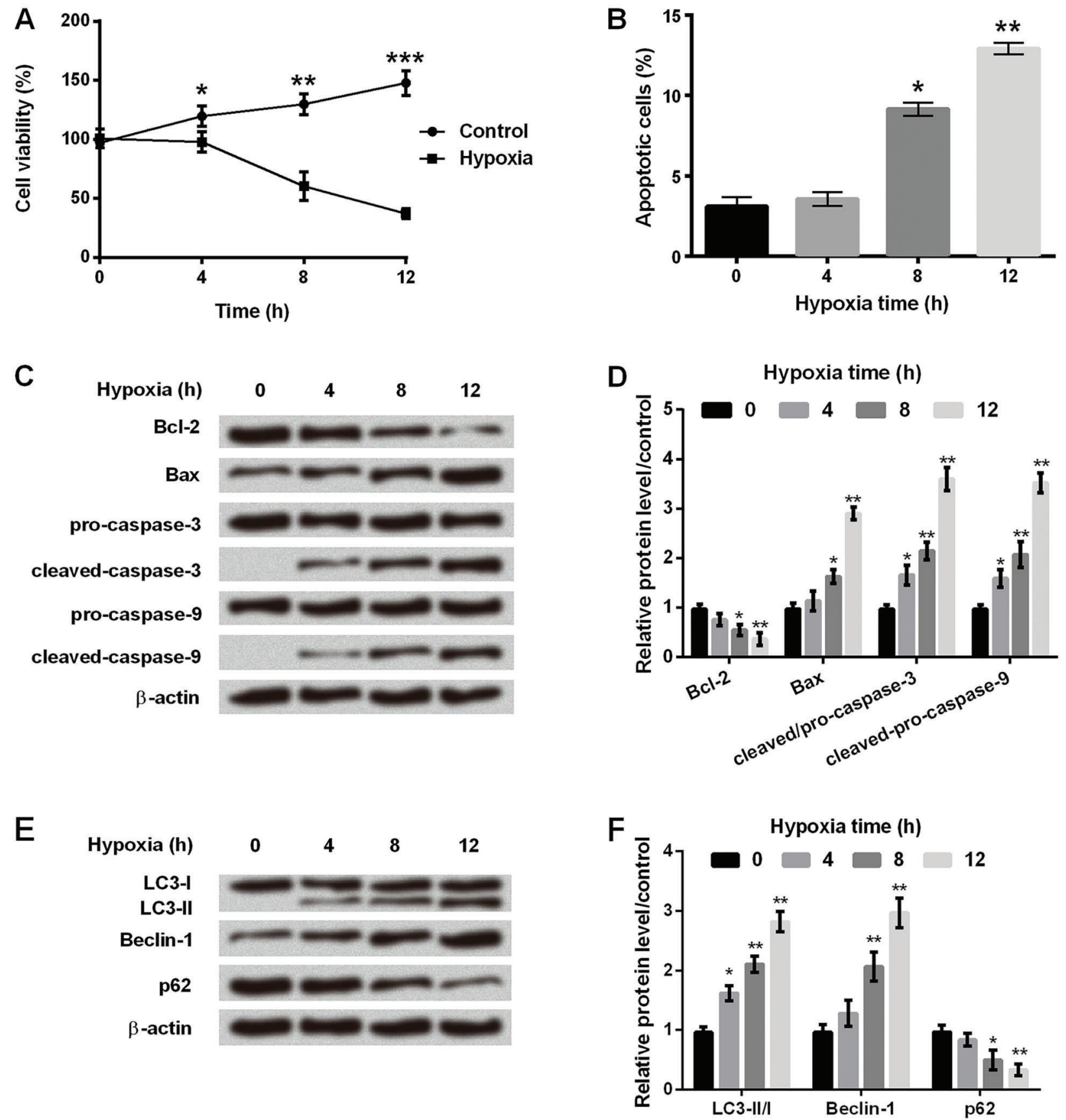

Figure 1. $A$, Cell viability was determined at different times in the control and hypoxia groups. $B$, Apoptosis rate was measured at different times in hypoxic astrocytes. $C$, Protein immunoblots of apoptosis-related factors by western blot assay. $D$, Relative expressions of apoptosis-related factors by quantification of band intensity. $E$, Protein immunoblots of autophagy-related factors by western blot assay. $F$, Relative expressions of autophagy-related factors by quantification of band intensity. $\beta$-actin acted as an internal control. Data are reported as means $\pm S D$. ${ }^{*} \mathrm{P}<0.05$, ${ }^{* \star} \mathrm{P}<0.01$, ${ }^{* \star *} \mathrm{P}<0.001$ compared to $0 \mathrm{~h}$ (ANOVA). 
Statistical analyses were performed using SPSS 22.0 statistical software (SPSS, USA) using a one-way analysis of variance (ANOVA). A P-value of $<0.05$ indicates a statistically significant result.

\section{Results}

Hypoxia affected cell viability, apoptosis, and autophagy of astrocytes

After culturing in hypoxia or control (normoxia) environment for 0 to $12 \mathrm{~h}, \mathrm{C} 8-\mathrm{D} 1 \mathrm{~A}$ cells were harvested and analyzed for cell viability, apoptosis, and autophagy. As shown in Figure $1 \mathrm{~A}$, cell viability was significantly reduced at 4 to $12 \mathrm{~h}(\mathrm{P}<0.05, \mathrm{P}<0.01$ or $\mathrm{P}<0.001)$ after hypoxia. Flow cytometry analysis demonstrated that the number of apoptotic cells was significantly increased after $8 \mathrm{~h}$ $(P<0.05)$ and $12 \mathrm{~h}(\mathrm{P}<0.01)$ in hypoxia (Figure $1 \mathrm{~B})$. Figure $1 \mathrm{C}$ and $\mathrm{D}$ showed that $\mathrm{Bcl}-2$ expression was significantly decreased, while Bax, cleaved-caspase-3, and cleaved-caspase- 9 expressions were significantly increased after $8 \mathrm{~h}$ in hypoxia incubator compared with control $(\mathrm{P}<0.05$ or $\mathrm{P}<0.01)$. We also analyzed expression of proteins involved in cell autophagy, and found that the ratio of LC3-II/LC-I and the amount of Beclin-1 were significantly increased, while that of p62 was decreased in a timedependent manner in hypoxia incubator compared to control (Figure $1 \mathrm{E}$ and $\mathrm{F}, \mathrm{P}<0.05$ or $\mathrm{P}<0.01$ ). These results suggested that cell apoptosis and autophagy were significantly increased after $8 \mathrm{~h}$ of hypoxic incubation in astrocytes.

\section{Hypoxia induced S100B expression in astrocytes}

Using qRT-PCR, we demonstrated that the mRNA amount of S100B was significantly increased after $8 \mathrm{~h}$ in hypoxia (Figure 2A, $\mathrm{P}<0.05$ ). Western blot also confirmed that protein expression of $\mathrm{S} 100 \mathrm{~B}$ was gradually increased in hypoxictreated astrocytes (Figure $2 \mathrm{~B}$ and $\mathrm{C}, \mathrm{P}<0.05$ or $\mathrm{P}<0.01$ ).

\section{ANDRO reduced cell apoptosis but promoted cell autophagy in hypoxic astrocytes}

To reveal the effect of ANDRO on apoptosis, firstly we used flow cytometry analysis. The number of apoptotic cells in hypoxic astrocytes was significantly decreased after adding ANDRO (Figure 3A, P<0.05). Secondly, we detected expression levels of proteins involved in apoptosis by western blot. As shown in Figure 3B and C, addition of ANDRO in hypoxic astrocytes resulted in an increase in Bcl-2 protein expression and decreases in Bax, cleaved-Caspase-3, and cleaved-Caspase- 9 protein expressions $(P<0.05$ or $P<0.01)$. These results suggested that ANDRO attenuates the process of apoptosis in hypoxic astrocytes. By analyzing expression of proteins related to cell autophagy after $12 \mathrm{~h}$ in hypoxia, we found that addition of ANDRO significantly promoted increases in LC3-II and Beclin-1 protein expressions, and a decline in p62 protein expression (Figure $3 D$ and $E, P<0.05, P<0.01$ or $P<0.001)$. This data showed that ANDRO promoted autophagy in hypoxic astrocytes.

\section{ANDRO promoted upregulation of $\mathrm{S} 100 \mathrm{~B}$ expression}

Both qRT-PCR (Figure 4A) and western blot analysis (Figure $4 \mathrm{~B}$ and $\mathrm{C}$ ) suggested that addition of ANDRO dramatically increased the expression of $\mathrm{S} 100 \mathrm{~B}$ in hypoxic astrocytes $(P<0.05$ or $P<0.01)$.

\section{ANDRO promoted autophagy and S100B expression by activating JNK signaling pathways}

Next, we investigated the underlying mechanisms through which ANDRO promoted autophagy and expression of $\mathrm{S} 100 \mathrm{~B}$. As shown in Figure $5 \mathrm{~A}$ and $\mathrm{B}$, adding ANDRO alone promoted expressions of $\mathrm{S} 100 \mathrm{~B}$ and autophagy-related proteins as described above. In addition, ANDRO increased the expressions of p-JNK and p-c-Jun. However, after treatment with JNK inhibitor, ANDRO treatment did not lead to increases in the expressions of LC3-II, Beclin-1, and $\mathrm{S} 100 \mathrm{~B}$, or a decline in p62 expression. These results suggested that the promotion of autophagy and S100B expression by ANDRO might be through activating JNK signaling pathways in hypoxic astrocytes.

\section{ANDRO promoted autophagy and S100B expression by upregulating expression of ATG5}

To reveal the regulation mechanism of ANDRO, we also demonstrated the association between ANDRO and
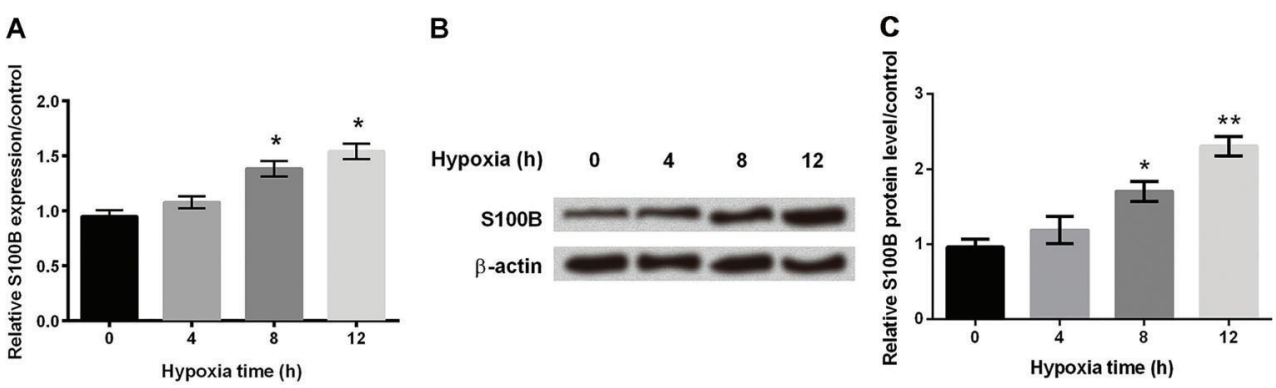

Figure 2. $A$, mRNA expression of $\mathrm{S} 100 \mathrm{~B}$ was determined by qRT-PCR at different times in hypoxic astrocytes. $B$ and $C$, Western blot was performed to assess the protein expression of $\mathrm{S} 100 \mathrm{~B}$ at different times in hypoxia astrocytes. $\beta$-actin acted as an internal control. Data are reported as means $\pm S D$. ${ }^{*} \mathrm{P}<0.05,{ }^{*} \mathrm{P}<0.01$ compared to $0 \mathrm{~h}$ (ANOVA). 

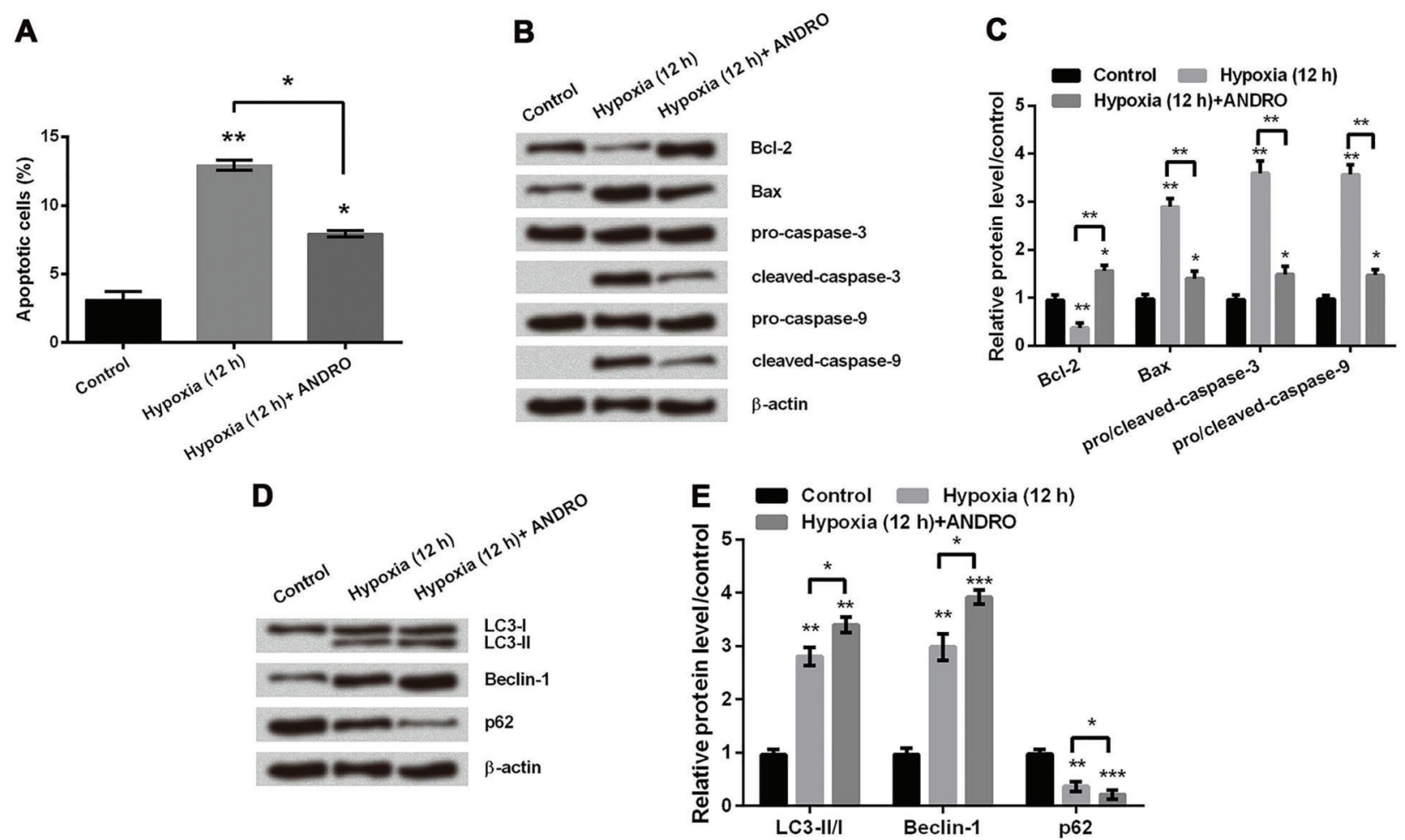

Figure 3. $A$, Apoptosis rate was measured in hypoxic astrocytes with or without treatment of andrographolide (ANDRO). $B$ and $C$, Western blot was used to determine the protein expression level of apoptosis related proteins in hypoxic astrocytes with or without treatment of ANDRO. $D$ and $E$, Protein expression of autophagy-regulated factors was determined by western blot. $\beta$-actin acted as an internal control. Data are reported as means $\pm \mathrm{SD}$. ${ }^{*} \mathrm{P}<0.05,{ }^{* \star} \mathrm{P}<0.01,{ }^{* * *} \mathrm{P}<0.001$ (ANOVA).
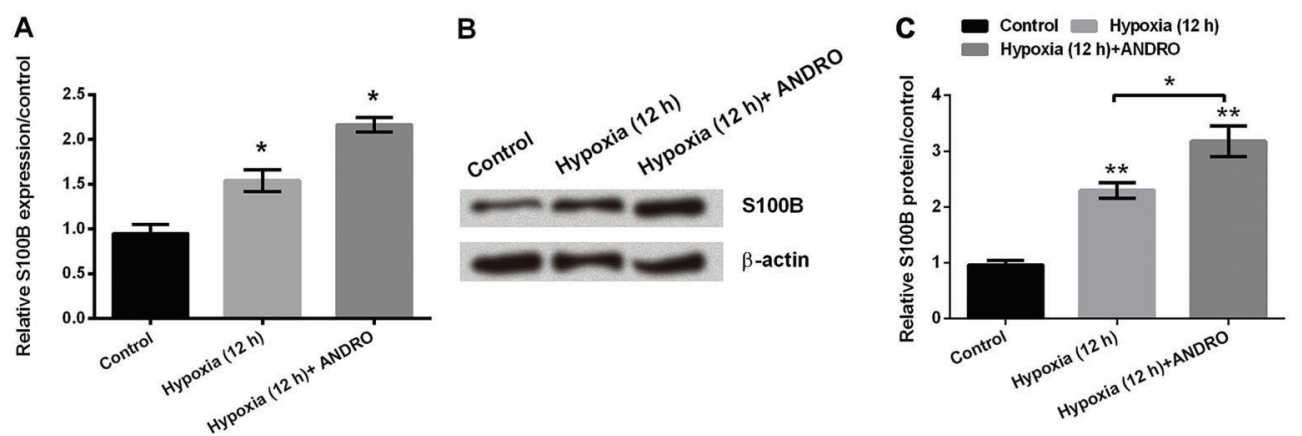

Figure 4. $A$, qRT-PCR was used to determine the mRNA expression of S100B in hypoxic astrocytes with or without treatment of andrographolide (ANDRO). $B$ and $C$, Western blot was performed to assess the protein expression of S100B in hypoxic astrocytes with or without treatment of ANDRO. $\beta$-actin acted as an internal control. Data are reported as means $\pm S D$. ${ }^{*} P<0.05$, ${ }^{\star *} P<0.01$ (ANOVA).

expressions of S100B and ATG5. Firstly, we found that expression of ATG5 was increased in hypoxic astrocytes with the treatment of ANDRO (Figure $6 A$ and $B, P<0.05$, $P<0.01$ or $P<0.001)$. Given the important role of ATG5 in autophagy process (24), this result provided more evidence that ANDRO promoted autophagy in hypoxic astrocytes. Secondly, when ATG5 was knocked down in hypoxic astrocytes with ANDRO treatment, expression of autophagy-related proteins (LC3-II, Beclin-1, and p62) were reduced, and interestingly the expression level of S100B was also reduced to that in control cells $(P<0.05$, $\mathrm{P}<0.01$ or $\mathrm{P}<0.001)$. Therefore, we concluded that ANDRO promoted $\mathrm{S} 100 \mathrm{~B}$ expression perhaps by upregulating expression of ATG5. 


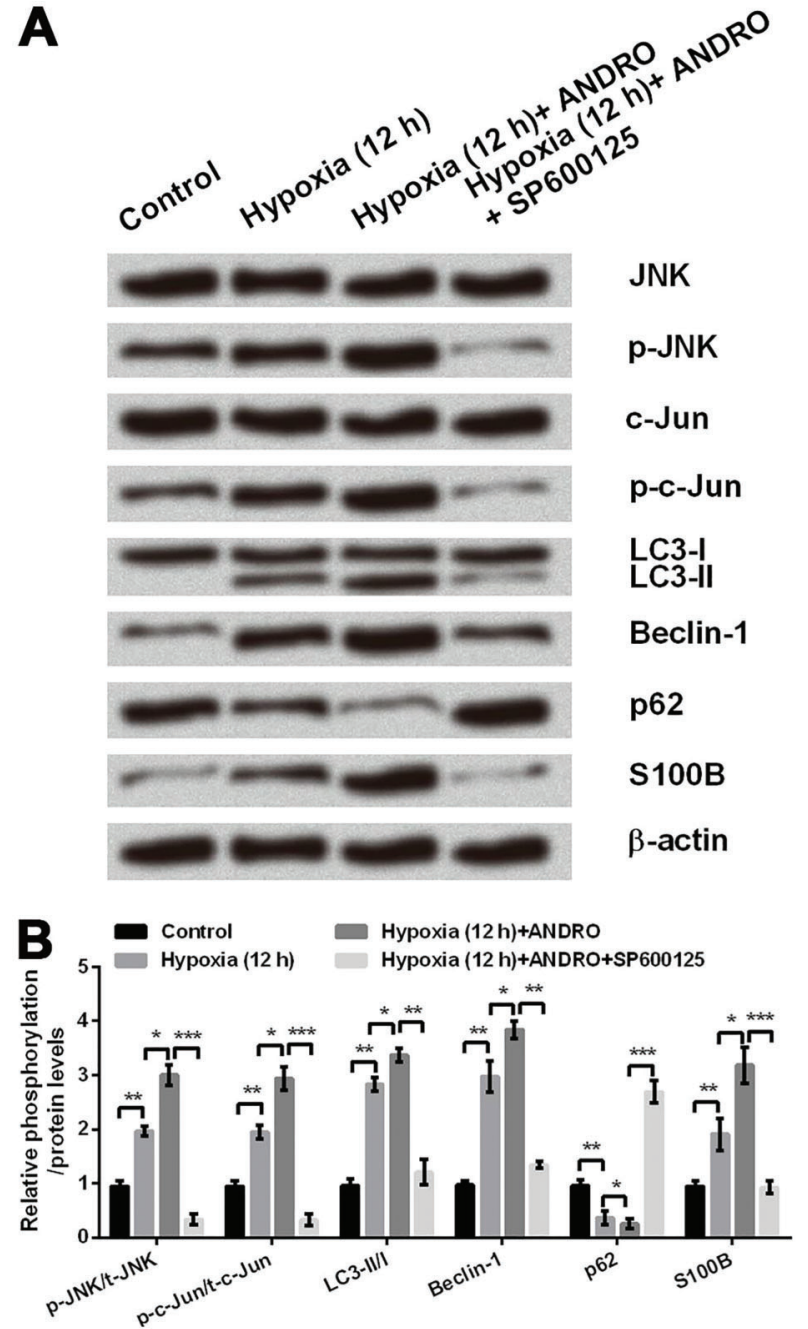

Figure 5. $A$, Protein immunoblots of $\mathrm{S} 100 \mathrm{~B}$ and proteins related to JNK pathway and autophagy measured by western blot in hypoxic astrocytes with or without treatment of andrographolide (ANDRO) and SP600125. B, Relative expressions of JNK pathway and autophagy related factors by quantification of band intensity. $\beta$-actin acted as an internal control. Data are reported as means $\pm \mathrm{SD}$. ${ }^{*} \mathrm{P}<0.05,{ }^{* *} \mathrm{P}<0.01,{ }^{* * *} \mathrm{P}<0.001$ (ANOVA).

\section{Discussion}

ANDRO has been well known for its various bioactivities such as anti-inflammatory (9). However, in hypoxia brain injury, the function of ANDRO has not been well studied. Recently ANDRO was reported to protect rat cardiomyocytes against hypoxia and reoxygenation injury and protect mice brain against hypoxia/ischemia injury $(19,20)$. Similarly, in the present study, we demonstrated that ANDRO protected mouse astrocytes against hypoxia injury.

To research the role of ANDRO in hypoxia-injured astrocytes, we investigated its effects on three aspects:
A
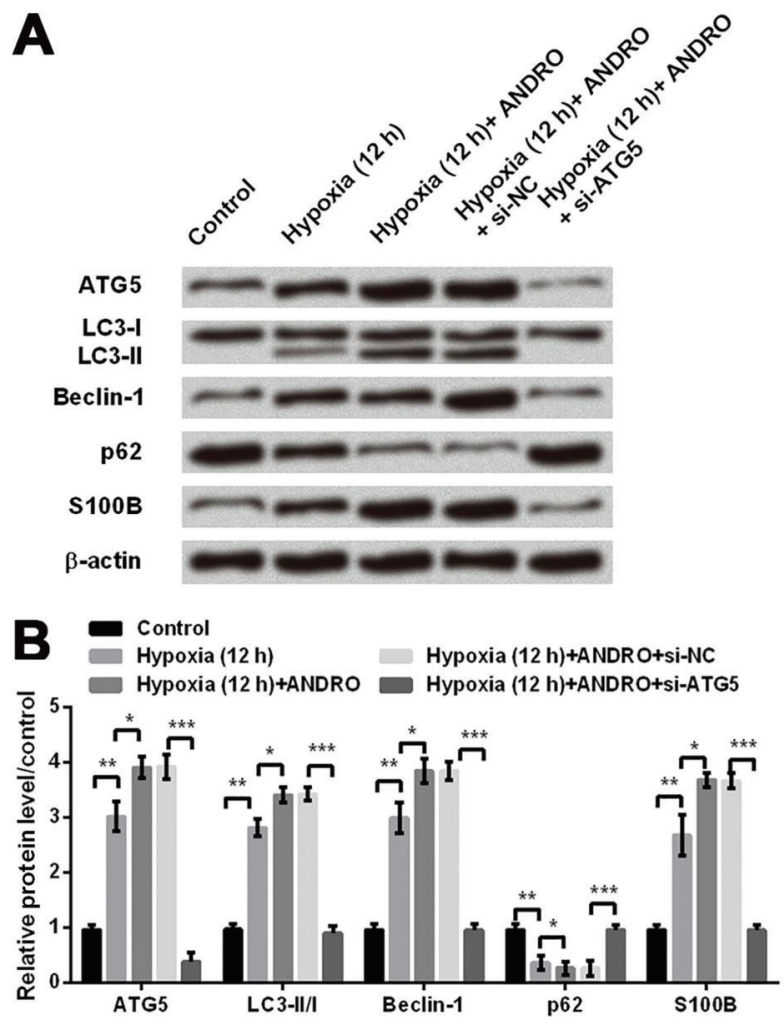

Figure 6. $A$, Protein immunoblots of ATG5, S100B, and autophagy-related proteins measured by western blot in hypoxic astrocytes with or without si-ATG5 transfection and treatment of andrographolide (ANDRO). B, Relative expressions of ATG5, $\mathrm{S} 100 \mathrm{~B}$ and autophagy-related proteins by quantification of band intensity. $\beta$-actin acted as an internal control. si-NC: non-targeting siRNA. Data are reported as means $\pm S D$. ${ }^{*} P<0.05$, ${ }^{*} P<0.01$, ${ }^{* * *} \mathrm{P}<0.001$ (ANOVA).

cell apoptosis, autophagy, and S100B expression. Flow cytometry analysis was performed to detect apoptotic cell rate, and expressions of a series of apoptosis-related proteins were measured. Caspases play an essential role in the transduction of apoptotic signals. When cytochrome $\mathrm{c}$ is released into the cytosol, it binds to an adaptor protein and pro-caspase-9, which in turn cleaves the procaspase- 9 into the active form (25). Caspase- 3 is activated by cleaved-caspase- 9 through proteolytic cleavage; then it degrades many intracellular proteins to carry out programed cell death (26). The Bcl-2 family, including anti-apoptotic (Bcl-2, Bcl-XL, Mcl-1) and proapoptotic members (Bid, Bax, Bad), is also one of the apoptotic regulatory proteins (27). Therefore, we used cleaved-caspase-3, cleaved-caspase-9, Bcl-2, and Bax as markers of apoptosis in our study. As a result, we found that the apoptotic cell rate and pro-apoptosis-related protein expressions were decreased and anti-apoptosis-related protein expression was increased by ANDRO, indicating 
that it attenuated the process of apoptosis in hypoxiainjured astrocytes.

Autophagy, also known as type II programmed cell death, causes orderly degradation and recycling of cellular components to survive bioenergetic stress (28). Beclin-1 is a component of the phosphatidylinositol-3-kinase complex that is required for autophagy (29). Beclin-1 expression has been recently used as a marker of autophagy $(30,31)$. During autophagy, there are two ATG5-dependent ubiquitin-like conjugation systems: the ATG12 conjugation system, leading to the formation of ATG12-ATG5-ATG16 molecular complexes, and the LC3 conjugation system, causing the LC3-I to generate a lipidated LC3-II form (24). LC3 also enables the docking of specific cargos and adaptor proteins such as p62 (Sequestosome-1)(32). Both conjugation systems lead to the formation of autophagosomes (24). Therefore, we used Beclin-1, LC3II, and p62 as markers of autophagy in our experiments. Results showed that expressions of Beclin-1 and LC3II were increased and p62 was decreased by ANDRO. Therefore, we concluded that ANDRO promoted autophagy in hypoxia-injured astrocytes. Many studies have shown that autophagy plays a protective role in brain injury $(32,33)$, and our results also confirm this point. Considering that ANDRO inhibited apoptosis while promoting autophagy, we can conclude that ANDRO induced cell autophagy from apoptosis to protect astrocytes from hypoxia damage.

S100B, produced mainly by astrocytes, exerts a neurotrophic effect and was shown to reduce brain injury $(34,35)$. In the present study, we found that the expression of S100B was increased by ANDRO in hypoxia-injured astrocytes.

\section{References}

1. Park E, Bell JD, Baker AJ. Traumatic brain injury: can the consequences be stopped? CMAJ 2008; 178: 1163-1170, doi: $10.1503 / \mathrm{cmaj} .080282$

2. Ghajar J. Traumatic brain injury. Kansas Nurse 2000; 356: 923-929.

3. Yu ACH, Gregory GA, Chan PH. Hypoxia-Induced Dysfunctions and injury of astrocytes in primary cell cultures. J Cereb Blood Flow Metab 1989; 9: 20-28, doi: 10.1038/ jcbfm.1989.3.

4. Oddo M, Levine JM, Mackenzie L, Frangos S, Feihl F, Kasner SE, et al. Brain hypoxia is associated with short-term outcome after severe traumatic brain injury independently of intracranial hypertension and low cerebral perfusion pressure. Neurosurgery 2011; 69: 1037, doi: 10.1227/NEU.0b01 3e3182287ca7.

5. Coon JT, Ernst E. Andrographis paniculata in the treatment of upper respiratory tract infections: a systematic review of safety and efficacy. Planta Med 2004; 70: 293-298, doi: 10.1055/s-2004-818938.

6. Jarukamjorn K, Nemoto N. Pharmacological Aspects of Andrographis paniculata on Health and Its Major Diterpenoid Constituent Andrographolide. J Health Sci 2008; 54: 370-381, doi: 10.1248/jhs.54.370.
Based on these findings, we inferred that ANDRO protected astrocytes against hypoxia injury by promoting S100B expression.

Next, in order to determine the mechanism responsible for regulation of autophagy and $\mathrm{S100B}$ expression by ANDRO, we tested the association between ANDRO, autophagy, and expression levels of S100B, JNK pathway proteins, and ATG5. Activated JNK regulates several important cellular functions including cell growth, differentiation, survival, and apoptosis by activating some small molecules such as c-Jun (36). Recently, JNK has been found to regulate autophagy $(37,38)$. In the current study, we found that ANDRO activated JNK pathway. When JNK pathway was inhibited, ANDRO could not upregulate autophagy and $\mathrm{S} 100 \mathrm{~B}$ expression as before. These results indicated that the promoting activities of ANDRO on autophagy and S100B expression might be through the JNK pathway. On the other hand, our study showed that knocking down ATG5 resulted in reduction of $\mathrm{S} 100 \mathrm{~B}$ expression in hypoxia-injured astrocytes with ANDRO treatment. This indicated that ATG5 reduced the expression of $\mathrm{S} 100 \mathrm{~B}$ even under the action of ANDRO. We, therefore, concluded that ANDRO promoted autophagy via the increased expression of S100B. However, this needs further research.

In conclusion, the present study indicated that ANDRO protected hypoxia-injured astrocytes against apoptosis and promoted autophagy and S100B expression. Our findings may have important implications in the treatment of hypoxia brain injury and application of ANDRO, although further research is still needed.
7. Chakravarti RN, Chakravarti D. Andrographolide, the active constituent of Andrographis paniculata Nees; a preliminary communication. Ind Med Gaz 1951; 86: 96.

8. Guan C, Min LI, Ren Q, Zhang W, Shan M, Wang R, et al. Andrographolide protects mice against severe Enterovirus 71 infection by its anti-inflammation and immunomodulation effects. Immunol J 2013; 29: 737-744.

9. Chen HW, Lin AH, Chu HC, Li CC, Tsai CW, Chao CY, et al. Inhibition of TNF- $\alpha$-Induced Inflammation by andrographolide via down-regulation of the PI3K/Akt signaling pathway. J Nat Prod 2011; 74: 2408-2413, doi: 10.1021/np200631v.

10. Rajagopal S, Kumar RA, Deevi DS, Satyanarayana C, Rajagopalan R. Andrographolide, a potential cancer therapeutic agent isolated from Andrographis paniculata. $J$ Exp Ther Oncol 2010; 3: 147-158, doi: 10.1046/j.1359-4117. 2003.01090.x.

11. Lin TP, Chen SY, Duh PD, Chang LK, Liu YN. Inhibition of the epstein-barr virus Iytic cycle by andrographolide. Biol Pharm Bull 2008; 31: 2018-2023, doi: 10.1248/bpb.31.2018.

12. Jiang $X, Y u$, Jiang J, Zhang Z, Wang Z, Yang Z, et al. Synthesis and evaluation of antibacterial activities of andrographolide analogues. Eur J Medi Chem 2009; 44: 2936, doi: 10.1016/j.ejmech.2008.12.014. 
13. Yu BC, Hung CR, Chen WC, Cheng JT. Antihyperglycemic effect of andrographolide in streptozotocin-induced diabetic rats. Planta Med 2003; 69: 1075-1079, doi: 10.1055/s-200345185.

14. Chan SJ, Wong WF, Wong PT, Bian JS. Neuroprotective effects of andrographolide in a rat model of permanent cerebral ischaemia. Br J Pharmacol 2010; 161: 668-679, doi: 10.1111/j.1476-5381.2010.00906.x.

15. Chen JH, Hsiao G, Lee AR, Wu CC, Yen MH. Andrographolide suppresses endothelial cell apoptosis via activation of phosphatidyl inositol-3-kinase/Akt pathway. Biochem Pharmacol 2004; 67: 1337-1345, doi: 10.1016/j.bcp.2003.12.015.

16. Burgos RA, Seguel K, Perez $M$, Meneses A, Ortega M, Guarda Ml, et al. Andrographolide inhibits IFN-gamma and IL-2 cytokine production and protects against cell apoptosis. Planta Med 2005; 71: 429-434, doi: 10.1055/s-2005-864138.

17. Kim TG, Hwi KK, Hung CS. Morphological and biochemical changes of andrographolide-induced cell death in human prostatic adenocarcinoma PC-3 cells. In Vivo 2005; 19: 551-557.

18. Zhao F, He EQ, Wang L, Liu K. Anti-tumor activities of andrographolide, a diterpene from Andrographis paniculata, by inducing apoptosis and inhibiting VEGF level. J Asian Nat Prod Res 2008; 10: 467-473, doi: 10.1080/102860208019 48334.

19. Woo AYH, Waye MMY, Tsui SKW, Yeung STW, Cheng CHK. Andrographolide Up-Regulates Cellular-Reduced Glutathione Level and Protects Cardiomyocytes against Hypoxia/Reoxygenation Injury. J Pharmacol Exp Ther 2008; 325: 226-235, doi: 10.1124/jpet.107.133918.

20. Chern CM, Liou KT, Wang YH, Liao JF, Yen JC, Shen YC. Andrographolide inhibits PI3K/AKT-dependent NOX2 and iNOS expression protecting mice against hypoxia/ischemiainduced oxidative brain injury. Planta Med 2011; 77: 16691679, doi: 10.1055/s-0030-1271019.

21. Harder DR, Zhang C, Gebremedhin D. Astrocytes function in matching blood flow to metabolic activity. Physiol 2002; 17: 27-31, doi: 10.1152/physiologyonline.2002.17.1.27.

22. Anderson MA, Burda JE, Ren $Y$, Ao Y, O'Shea TM, Kawaguchi $R$, et al. Astrocyte scar formation aids central nervous system axon regeneration. Nature 2016; 532: 195, doi: 10.1038/nature17623.

23. Walz W. Role of astrocytes in the clearance of excess extracellular potassium. Neurochem Int 2000; 36: 291-300, doi: 10.1016/S0197-0186(99)00137-0.

24. Joubert PE, Meiffren G, Grégoire IP, Pontini G, Richetta C, Flacher $M$, et al. Autophagy induction by the pathogen receptor CD46. Cell Host Microbe 2009; 6: 354-366, doi: 10.1016/j.chom.2009.09.006.

25. Bratton SB, Salvesen GS. Regulation of the Apaf-1-caspase9 apoptosome. J Cell Sci 2010; 123: 3209-3214, doi: 10.1242/ jcs.073643.
26. Riedl SJ, Shi Y. Molecular mechanisms of caspase regulation during apoptosis. Nat Rev Mol Cell Biol 2004; 5: 897907, doi: 10.1038/nrm1496.

27. Cory S, Adams JM. The Bcl2 family: regulators of the cellular life-or-death switch. Nat Rev Cancer 2002; 2: 647, doi: 10.1038/nrc883.

28. Mizushima N, Komatsu M. Autophagy: renovation of cells and tissues. Cell 2011; 147: 728-741, doi: 10.1016/j.cell. 2011.10.026.

29. Itakura E, Kishi C, Inoue K, Mizushima N. Beclin 1 forms two distinct phosphatidylinositol 3-kinase complexes with mammalian Atg14 and UVRAG. Mol Biol Cell 2008; 19: 53605372, doi: 10.1091/mbc.E08-01-0080.

30. Rami A, Langhagen A, Steiger S. Focal cerebral ischemia induces upregulation of Beclin 1 and autophagy-like cell death. Neurobiol Dis 2008; 29: 132-141, doi: 10.1016/j.nbd. 2007.08.005.

31. Carloni S, Buonocore G, Balduini W. Protective role of autophagy in neonatal hypoxia-ischemia induced brain injury. Neurobiol Dis 2008; 32: 329-339, doi: 10.1016/j.nbd.2008. 07.022 .

32. Sungwoo P, Seon-Guk C, Seung-Min Y, Jin H, Son, YongKeun J. Choline dehydrogenase interacts with SQSTM1/p62 to recruit LC3 and stimulate mitophagy. Autophagy 2014; 10 : 1906-1920, doi: 10.4161/auto.32177.

33. Balduini W, Carloni S, Buonocore G. Autophagy in hypoxiaischemia induced brain injury. J Matern Fetal Neonatal Med 2012; 25 (Suppl 1): 30, doi: 10.3109/14767058.2012. 663176.

34. Willoughby KA, Kleindienst A, Müller C, Chen T, Muir JK, Ellis EF. S100B protein is released by in vitro trauma and reduces delayed neuronal injury. J Neurochem 2010; 91: 1284-1291, doi: 10.1111/j.1471-4159.2004.02812.x.

35. Ellis EF, Willoughby KA, Sparks SA, Chen T. S100B protein is released from rat neonatal neurons, astrocytes, and microglia by invitro trauma and anti-S100 increases trauma-induced delayed neuronal injury and negates the protective effect of exogenous S100B on neurons. J Neurochem 2007; 101: 1463-1470, doi: 10.1111/j.1471-4159.2007. 04515.x.

36. Davis RJ. Signal transduction by the JNK group of MAP kinases. Cell 2000; 103: 239-252, doi: 10.1016/S0092-8674 (00)00116-1.

37. Lorin S, Pierron G, Ryan KM, Codogno P, Djavaheri-Mergny $M$. Evidence for the interplay between JNK and p53-DRAM signalling pathways in the regulation of autophagy. Autophagy 2010; 6: 153, doi: 10.4161/auto.6.1.10537.

38. Xie CM, Chan WY, Yu S, Zhao J, Cheng CH. Bufalin induces autophagy-mediated cell death in human colon cancer cells through reactive oxygen species generation and JNK activation. Free Radic Biol Med 2011; 51: 1365-1375, doi: 10.1016/ j.freeradbiomed.2011.06.016. 